\title{
Ephemeroptera, Plecoptera and Trichoptera in springs in Trentino (south-eastern Alps)
}

\author{
Bruno MAIOLINI*, Mauro CAROLLI and Luana SILVERI \\ Department of Invertebrate Zoology and Hydrobiology, Museo Tridentino di Scienze Naturali, Via Calepina 14, 38122 Trento, Italy \\ Present address: IASMA Research and Innovation Centre, Fondazione Edmund Mach, Environment and Natural Resources Area. Via \\ E. Mach 1, 38010 S. Michele all'Adige (TN), Italy \\ *e-mail corresponding author: bruno.maiolini@iasma.it
}

\begin{abstract}
Within the CRENODAT project (Biodiversity assessment and integrity evaluation of springs of Trentino - Italian Alps - and longterm ecological research, 2004-2008) we studied a total of 90 springs in Trentino (south-eastern Alps, Italy), 75 of which were used for statistical analysis. The springs were grouped into seven different types and represented all the available lithologies in the study area. Macrozoobenthos (Ephemeroptera, Plecoptera and Trichoptera; EPT) was collected from stones, bryophytes and sediments. We investigated which physical, chemical or environmental features were important in determining EPT assemblage metrics at sites, by calculating the Shannon-Wiener diversity index, and applying a one-way ANOVA test, ANOSIM and SIMPER analyses. Statistical results highlighted the island character of the springs, each spring having its specific history and abiotic characteristics, which select for unique community patterns. For the faunistic analyses, we considered all springs where EPT taxa were recorded; a total of 88 taxa. Highest species richness was recorded among the Trichoptera, followed by the Plecoptera and Ephemeroptera, with 52, 30 and 6 taxa respectively. Fifteen Trichoptera and three Plecoptera species were recorded as new for the Autonomous Province of Trento. Our results confirm that, in the harsh environment of the Alps, the mild and stable ecological conditions that characterize spring-fed brooks contribute to maintaining and enhancing the regional biodiversity. Springs act as refuge areas for stream biota, providing more favourable conditions during spates or droughts (common in Alpine headwaters), or for particular stages of the insect life cycles. Springs also provide specific habitats for strictly crenobiontic species.
\end{abstract}

Key words: freshwater ecology, macroinvertebrates, benthos, crenon, headwaters

\section{INTRODUCTION}

The specific ecological signature of springs is their constant physico-chemical characteristics (Glazier 1998; Williams \& Williams 1998). Because springs are located at the interface between two distinct ecosystems (ground-water and surface-water) they create a mosaic of aquatic and semi-aquatic microhabitats, and can be considered as "hot spots" for aquatic biodiversity (Cantonati et al. 2006; Staudacher \& Füreder 2007). The macroinvertebrate composition of springs can be influenced by various environmental, physical and chemical factors, such as water chemistry (Glazier 1991; Orendt 2000), water current velocity (Ilmonen \& Paasivirta 2005; von Fumetti et al. 2006), substratum composition (Glazier \& Gooch 1987; Hahn 2000) or altitude (Barquín \& Death 2006).

In the Alps, springs represent a habitat that is less affected by natural environmental disturbance (floods, droughts, extreme temperatures) than headwater streams (Staudacher \& Füreder 2007). Consequently they make an important contribution to the regional biodiversity of freshwater ecosystems (Ward \& Tockner 2001; Ilmonen et al. 2009) and can be considered as patchy habitat islands (Myers \& Resh 2002).

In Italy, faunistical studies have been conducted mainly in north-eastern Alpine and pre-Alpine springs
(Crema et al. 1996; Gerecke et al. 1998; Cantonati 1998; Stoch \& Tomasin 2002; Mezzanotte \& Sambugar 2004; Cantonati et al. 2006; Sambugar et al. 2006; Stoch et al. 2008) and in central and southern Apennine areas (Galassi 1997; Cianficconi et al. 1998; D'Ambrosio et al. 2003; Di Lorenzo et al. 2003; Di Sabatino et al. 2003; Bottazzi et al. 2008).

This study focused on the Ephemeroptera, Plecoptera and Trichoptera (EPT) communities of Alpine springs on the Italian north eastern Alps. The aim was to assess the faunal composition of selected springs, in particular (1) to analyse the distribution patterns of distinct EPT taxa; (2) to assess the EPT assemblages of the springs in the study area; and (3) to assess which physical, chemical or environmental factors are important in determining EPT assemblages and the species richness of each spring.

\section{METHODS}

\subsection{Study Area}

The study area is located in Trentino (south-eastern Alps, Italy). A total of 108 springs were chosen as a representative subset of the area and sampled. The selected springs covered a wide altitudinal range (from $170 \mathrm{~m}$ a.s.l. to $2792 \mathrm{~m}$ a.s.1.) (Fig. 1) and their locations are representative of the various lithologies of Trentino: from limestones and dolomites to siliceous substrata. 


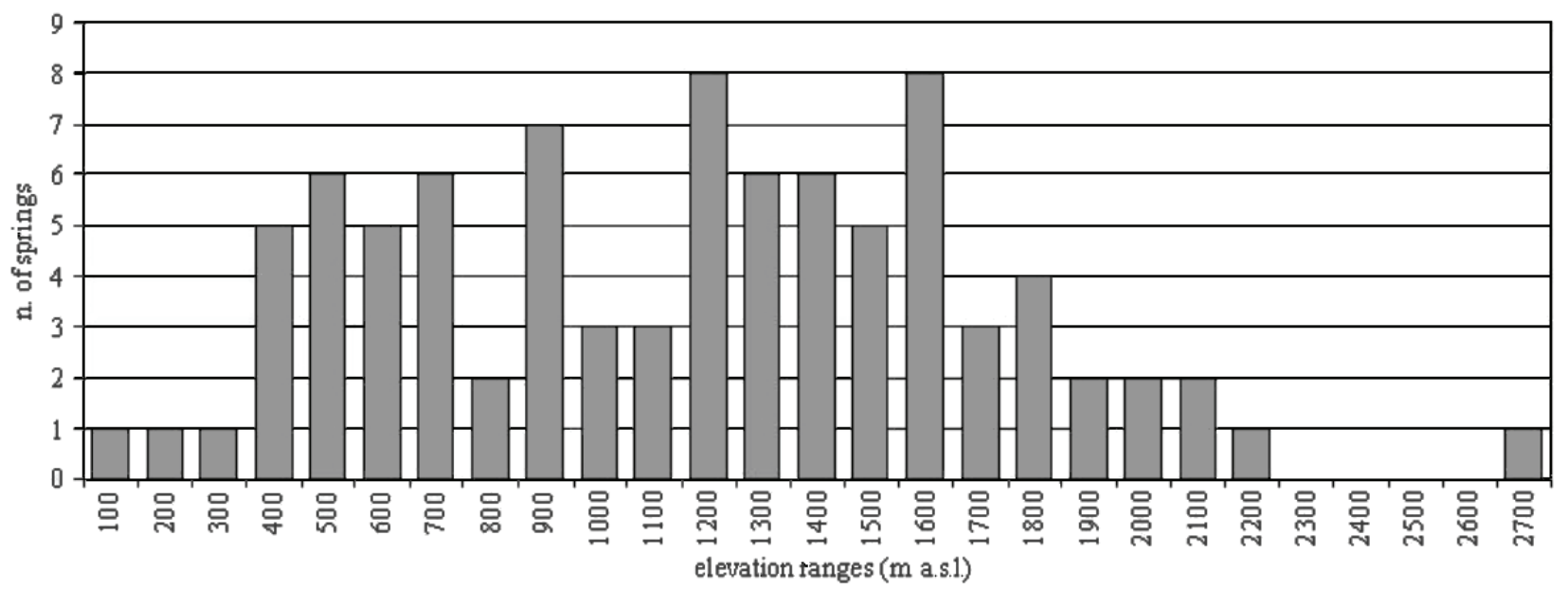

Fig. 1. Number of springs per elevation range, e.g. $100=100-199 ; 200=200-299$ etc.

For a complete description of the study area and of the environmental dataset see Cantonati et al. (2005).

\subsection{Data collection}

EPT taxa were collected in 99 of the 108 springs, but specimens were identified with enough confidence only for the material from 88 sites (due to the presence of only juvenile stages in some samples) and from the two long-term study springs.

Macrozoobenthos was collected separately from stones, bryophytes and sediments in the eucrenal zone of each spring. Specimens living on stones were collected with a $100 \mu \mathrm{m}$ mesh-size net, disturbing a $0.06 \mathrm{~m}^{2}$ area. About $10 \mathrm{~g}$ of bryophytes and $50 \mathrm{~mL}$ of surface sediments were collected and the samples were sorted in the laboratory. Organisms were expressed as number of individuals per $\mathrm{m}^{2}$. Samples were collected for each spring once in the summer season of 2005 and 2006, with the exception of two springs which are part of a long term monitoring project.

A detailed description of the sampling methods is presented in Cantonati et al. (2007) Gerecke et al. (2011, this issue) and in Spitale et al. (2011, submitted).

Specimens were identified to species level according to Lechthaler \& Stockinger (2005), Waringer \& Graf (1997) and Moretti (1983) for Trichoptera, Fochetti \& Tierno de Figueroa (2008) and Consiglio (1980) for Plecoptera, and Belfiore (1983) and Sartori \& Landolt (1999) for Ephemeroptera. Adult specimens were identified when available.

\subsection{Data processing and statistical analysis}

In order to be able to compare with other results (Spitale et al. 2011, submitted), we ran the statistical analyses using only the 75 springs with identifiable EPT taxa. Non-hierarchical cluster analyses PAM (Partitioning Around Medoids) was applied to the environmental variables dataset to generate the spring types (Spitale et al. 2011, submitted). Springs were thus divided into 7 groups: rheocrene springs at low altitude (RhLA), rheocrene springs at high altitude (RhHA), rheocrene springs on siliceous substratum (RhS), large rheocrene springs (RhL), hygropetric springs (Hyg), helocrene springs (Hel), and limnocrene springs (Lim). In particular, according to the analyses performed by Spitale et al. (2011, submitted), RhLA differed from RhHA in altitude (the latter were mainly located at higher elevations), in shading percentage (higher in RhLA), current velocity and discharge (higher in RhHA). Rheocrenes on siliceous substrata (RhS) differed from RhLA and RhHA in lower $\mathrm{pH}$ and lower degree of shading. $\mathrm{RhS}$ were also the group located at highest altitudes. Large rheocrene springs $(\mathrm{RhL})$ were characterized by higher discharge and current velocity, whilst hygropetric rheocrenes (springs with a thin water film flowing over the bedrock, Hyg) presented low discharge but a relatively high velocity. Finally, helocrenes (Hel) and limnocrenes (Lim) differed from all other spring types in substratum composition (sand and clay in these two groups) and from each other by water velocity (Spitale et al. 2011, submitted). Lim differed from other spring types, except for RhL, also in higher discharge, while Hel showed the highest percentage of organic substratum.

This classification used a large number of environmental variables and was therefore used as a template to detect possible distributional patterns in Trentino EPT crenal communities.

All springs were sampled in 2005 (Tab. 1), four were also sampled in 2006 and long-term data were available for two, but we chose to perform the statistical analysis using only the data from 2005 , considering one sample for each site, due to the lack of a complete dataset for 2006.

As a consequence, only 69 of the total 88 identified taxa were considered in the statistical analyses, while the whole species list was used for faunistic considerations. 
Tab. 1. List of the 90 springs with EPT taxa, numbers represent species as listed in table 2. Sampling dates are presented for 2005. Besides the two long-term monitored springs, AD2153, LD0509, SC0250 (29-0/8) and BR1315 (6/5;31/5 and 13/11) were also sampled in 2006. Elevation (m a.s.1.) is shown by the numeric part of the spring names. Lithology as in Cantonati et al. (2007).

\begin{tabular}{|c|c|c|c|c|}
\hline Spring & EPT species assemblage & 2005 sampling dates & Mountain Group & Lithology \\
\hline AD0905 & $18 ; 35 ; 61 ; 80 ; 81$ & $11 / 7$ & Adamello-Presanella & $\mathrm{Ca}-\mathrm{Cr}$ \\
\hline AD1077 & $2 ; 9 ; 10 ; 30 ; 38 ; 47 ; 70 ; 76$ & $11 / 7$ & Adamello-Presanella & $\mathrm{Ca}$ \\
\hline AD1235 & $47 ; 76$ & $16 / 8$ & Adamello-Presanella & $\mathrm{Ca}$ \\
\hline AD1300 & $\begin{array}{l}1 ; 2 ; 3 ; 4 ; 5 ; 10 ; 13 ; 14 ; 15 ; 16 ; 17 ; 19 ; 23 ; 25 ; 27 ; 29 ; 30 \\
40 ; 41 ; 42 ; 47 ; 56 ; 57 ; 58 ; 67 ; 68 ; 72 ; 73 ; 76 ; 80 ; 83\end{array}$ & long-term monitoring & Adamello-Presanella & $\mathrm{Cr}$ \\
\hline AD1353 & $23 ; 42 ; 51$ & $11 / 10$ & Adamello-Presanella & $\mathrm{Cr}$ \\
\hline AD1654 & $10 ; 14 ; 25 ; 34$ & $16 / 8$ & Adamello-Presanella & $\mathrm{Ca}$ \\
\hline AD1790 & $51 ; 69$ & $26 / 10$ & Adamello-Presanella & $\mathrm{Cr}$ \\
\hline AD1990 & $17 ; 25 ; 29 ; 34 ; 42 ; 52 ;$ & $1 / 9$ & Adamello-Presanella & $\mathrm{Cr}$ \\
\hline AD2153 & $2 ; 4 ; 5 ; 6 ; 25 ; 27 ; 31 ; 35 ; 37 ; 41 ; 51 ; 57 ; 60$ & $25 / 5 ; 24 / 10$ & Adamello-Presanella & $\mathrm{Cr}$ \\
\hline AN0430 & $44 ; 61$ & $27 / 6$ & Anauni & $\mathrm{Ca}-\mathrm{Cr}$ \\
\hline AN0590 & $14 ; 61 ; 81$ & $21 / 7$ & Anauni & $\mathrm{Ca}$ \\
\hline AN1474 & $2 ; 14 ; 17 ; 24 ; 25 ; 27 ; 68 ; 83$ & $21 / 7$ & Anauni & $\mathrm{Ca}$ \\
\hline AN1578 & $23 ; 27$ & $17 / 10$ & Anauni & $\mathrm{Cr}$ \\
\hline AN1685 & $17 ; 23 ; 27 ; 50 ; 52 ; 53 ; 57$ & $18 / 8$ & Anauni & $\mathrm{Cr}$ \\
\hline AN1950 & $6 ; 57 ; 61$ & $27 / 6 ; 2 / 9$ & Anauni & $\mathrm{Cr}$ \\
\hline AT0756 & $1 ; 3 ; 25 ; 27 ; 30 ; 47$ & $29 / 6$ & Vigolana & $\mathrm{Ca}$ \\
\hline AT0972 & $3 ; 11 ; 14 ; 25 ; 47 ; 61$ & $15 / 7 ; 15 / 9$ & Vigolana & $\mathrm{Ca}$ \\
\hline AT1052 & $41 ; 58$ & $11 / 8$ & Vigolana & $\mathrm{Ca}$ \\
\hline ВC0170 & $2 ; 3 ; 6 ; 11 ; 61$ & $13 / 6$ & Brento-Casale & $\mathrm{Ca}$ \\
\hline ВC0503 & 44 & 9/7 & Brento-Casale & $\mathrm{Ca}$ \\
\hline ВC0565 & $3 ; 66 ; 75 ; 80 ; 81$ & $12 / 7$ & Brento-Casale & $\mathrm{Ca}$ \\
\hline BR0470 & $1 ; 2 ; 4 ; 5 ; 31 ; 35 ; 74$ & $13 / 7$ & Brenta & $\mathrm{Ca}$ \\
\hline BR0510 & $3 ; 7 ; 14 ; 23 ; 25 ; 27 ; 28 ; 44 ; 47 ; 66 ; 88$ & $22 / 6$ & Brenta & $\mathrm{Ca}$ \\
\hline BR0658 & $3 ; 7 ; 27 ; 47 ; 61 ; 66 ; 77 ; 81$ & $1 / 8$ & Brenta & $\mathrm{Ca}-\mathrm{Cr}$ \\
\hline BR0679 & $1 ; 12 ; 25 ; 35$ & $3 / 8$ & Brenta & $\mathrm{Ca}$ \\
\hline BR0686 & $35 ; 36$ & $29 / 11$ & Brenta & $\mathrm{Ca}$ \\
\hline BR0735 & $14 ; 18 ; 81$ & $2 / 8$ & Brenta & $\mathrm{Ca}$ \\
\hline BR0790 & $1 ; 2 ; 14 ; 27 ; 47 ; 77 ; 81$ & $12 / 7$ & Brenta & $\mathrm{Ca}$ \\
\hline BR0804 & $14 ; 24 ; 27 ; 41 ; 82$ & $22 / 7$ & Brenta & $\mathrm{Cr}$ \\
\hline BR0950 & $25 ; 27 ; 57$ & $12 / 7$ & Brenta & $\mathrm{Ca}-\mathrm{Cr}$ \\
\hline BR1315 & $3 ; 23 ; 25 ; 28 ; 45 ; 56 ; 57 ; 65 ; 76 ; 83$ & $31 / 5$ & Brenta & $\mathrm{Ca}$ \\
\hline BR1358 & $\begin{array}{l}1 ; 3 ; 4 ; 9 ; 10 ; 12 ; 14 ; 15 ; 17 ; 20 ; 21 ; 25 ; 29 ; 31 ; 35 \\
41 ; 46 ; 48 ; 49 ; 51 ; 58 ; 59 ; 71 ; 74 ; 75 ; 76 ; 79 ; 80\end{array}$ & long-term monitoring & Brenta & $\mathrm{Ca}$ \\
\hline BR1379 & $12 ; 17 ; 27 ; 35 ; 52 ; 57 ; 68$ & $22 / 9 ; 4 / 11$ & Brenta & $\mathrm{Ca}$ \\
\hline BR1436 & $24 ; 27 ; 78$ & $17 / 8$ & Brenta & $\mathrm{Ca}$ \\
\hline BR1605 & $14 ; 22 ; 23 ; 25 ; 76 ; 79 ; 86$ & $17 / 8$ & Brenta & $\mathrm{Ca}$ \\
\hline BR1765 & 27 & $26 / 10$ & Brenta & $\mathrm{Ca}$ \\
\hline BR2240 & 24 & $10 / 10$ & Brenta & $\mathrm{Ca}$ \\
\hline BS0420 & $27 ; 39$ & $29 / 7$ & Bondone-Stivo & $\mathrm{Ca}$ \\
\hline BS0705 & $14 ; 27 ; 47 ; 66 ; 77$ & $8 / 7$ & Bondone-Stivo & $\mathrm{Ca}$ \\
\hline BS1527 & $6 ; 24 ; 66$ & $24 / 8$ & Bondone-Stivo & $\mathrm{Ca}$ \\
\hline CA1642 & $17 ; 25$ & $15 / 9$ & Lagorai-Cima d'Asta & $\mathrm{Cr}$ \\
\hline CA2153 & $43 ; 54 ; 76$ & $14 / 9$ & Lagorai-Cima d'Asta & $\mathrm{Cr}$ \\
\hline CS1350 & $24 ; 30 ; 52 ; 62$ & $9 / 8$ & Catinaccio & $\mathrm{Ca}$ \\
\hline CV0250 & $25 ; 42$ & $28 / 6$ & Lagorai-Cima d'Asta & $\mathrm{Cr}$ \\
\hline CV0633 & $27 ; 67 ; 82$ & $4 / 7$ & Lagorai-Cima d'Asta & $\mathrm{Cr}$ \\
\hline CV0854 & $14 ; 61 ; 68$ & $5 / 7$ & Lagorai-Cima d'Asta & $\mathrm{Cr}$ \\
\hline CV0962 & $14 ; 25 ; 57 ; 64$ & $8 / 8$ & Lagorai-Cima d'Asta & $\mathrm{Cr}$ \\
\hline CV0992 & $1 ; 2 ; 3 ; 17 ; 27 ; 30 ; 34 ; 35 ; 80$ & $20 / 7$ & Lagorai-Cima d'Asta & $\mathrm{Cr}$ \\
\hline CV1084 & $23 ; 25 ; 27 ; 34 ; 47$ & $27 / 7$ & Lagorai-Cima d'Asta & $\mathrm{Ca}-\mathrm{Cr}$ \\
\hline CV1200 & $27 ; 35 ; 54 ; 57 ; 63 ; 79$ & $19 / 7$ & Lagorai-Cima d'Asta & $\mathrm{Cr}$ \\
\hline CV1215 & $25 ; 27 ; 41 ; 68$ & $8 / 8$ & Lagorai-Cima d'Asta & $\mathrm{Cr}$ \\
\hline CV1254 & $24 ; 32 ; 35 ; 43$ & $8 / 8$ & Lagorai-Cima d'Asta & $\mathrm{Cr}$ \\
\hline CV1280 & $12 ; 47 ; 57 ; 83$ & $10 / 8$ & Lagorai-Cima d'Asta & $\mathrm{Cr}$ \\
\hline CV1421 & $25 ; 42 ; 44 ; 57 ; 85$ & $21 / 9$ & Lagorai-Cima d'Asta & $\mathrm{Cr}$ \\
\hline CV1435 & $2 ; 17 ; 23 ; 26 ; 27 ; 34 ; 35 ; 41 ; 51 ; 57 ; 68$ & $28 / 7$ & Lagorai-Cima d'Asta & $\mathrm{Cr}$ \\
\hline CV1575 & 28 & $21 / 9$ & Lagorai-Cima d'Asta & $\mathrm{Cr}$ \\
\hline CV1623 & $2 ; 14 ; 41$ & $18 / 8$ & Lagorai-Cima d'Asta & $\mathrm{Cr}$ \\
\hline CV1655 & $10 ; 57 ; 76$ & $22 / 9$ & Lagorai-Cima d'Asta & $\mathrm{Cr}$ \\
\hline CV1685 & $25 ; 57 ; 68$ & $23 / 8$ & Lagorai-Cima d'Asta & $\mathrm{Cr}$ \\
\hline CV1855 & $2 ; 5 ; 17 ; 41 ; 57$ & $19 / 8$ & Lagorai-Cima d'Asta & $\mathrm{Cr}$ \\
\hline
\end{tabular}

(continued) 
Tab. 1. Continuation.

\begin{tabular}{llccc}
\hline Spring & EPT species assemblage & 2005 sampling dates & Mountain Group & Lithology \\
\hline CV2051 & $5 ; 54$ & $23 / 8$ & Lagorai-Cima d'Asta & $\mathrm{Cr}$ \\
CV2126 & $24 ; 25$ & $21 / 9$ & Lagorai-Cima d'Asta & $\mathrm{Cr}$ \\
LD0420 & $7 ; 14 ; 27 ; 55$ & $21 / 6$ & Alpi di Ledro & $\mathrm{Ca}$ \\
LD0509 & $14 ; 27 ; 28 ; 44 ; 45 ; 47 ; 81$ & $6 / 7$ & Alpi di Ledro & $\mathrm{Ca}$ \\
LD0584 & $8 ; 14 ; 25 ; 35 ; 58 ; 81$ & $6 / 7$ & Alpi di Ledro & $\mathrm{Ca}$ \\
LD0720 & $14 ; 17 ; 27 ; 81$ & $7 / 9$ & Alpi di Ledro & $\mathrm{Ca}$ \\
LD0930 & $25 ; 27 ; 30 ; 45 ; 76 ; 87$ & $29 / 6$ & Alpi di Ledro & $\mathrm{Ca}$ \\
LD1160 & $10 ; 24 ; 25 ; 27 ; 76 ; 84$ & $26 / 7$ & Alpi di Ledro & $\mathrm{Ca}$ \\
LD1400 & $3 ; 14 ; 41$ & $26 / 7$ & Alpi di Ledro & $\mathrm{Ca}$ \\
LD1502 & $23 ; 41$ & $17 / 7$ & Alpi di Ledro & $\mathrm{Ca}$ \\
LT1240 & $1 ; 27 ; 35 ; 44 ; 47 ; 57 ; 70 ; 77$ & $18 / 7$ & Latemar & $\mathrm{Ca}-\mathrm{Cr}$ \\
MB0335 & 1 & $15 / 6$ & Monte Baldo & $\mathrm{Ca}$ \\
MB1440 & 57 & $2 / 11$ & Monte Baldo & $\mathrm{Ca}$ \\
MC1115 & $24 ; 35 ; 47 ; 61$ & $12 / 8$ & Monte Corona & $\mathrm{Ca}$ \\
MD1670 & 37 & $26 / 9$ & Marmolada & $\mathrm{Ca}-\mathrm{Cr}$ \\
MD1871 & $24 ; 27 ; 35 ; 43 ; 77$ & $26 / 9$ & Marmolada & $\mathrm{Ca}$ \\
MP0656 & $3 ; 12 ; 54 ; 83 ; 84$ & $25 / 7$ & Pasubio & $\mathrm{Ca}$ \\
MP1566 & 27 & $2 / 11$ & Pasubio & $\mathrm{Ca}$ \\
OC0981 & 65 & $22 / 7$ & Ortles-Cevedale & $\mathrm{Cr}$ \\
OC2056 & $1 ; 35 ; 80$ & $4 / 11$ & Ortles-Cevedale & $\mathrm{Cr}$ \\
OC2792 & $37 ; 51 ; 56 ; 68$ & $27 / 9$ & Ortles-Cevedale & $\mathrm{Cr}$ \\
PG0453 & $11 ; 25 ; 27 ; 35 ; 47 ; 61$ & $27 / 6$ & Paganella-Gazza & $\mathrm{Ca}$ \\
PG0474 & $2 ; 3 ; 34 ; 42 ; 61 ; 81 ; 83$ & $14 / 6$ & Paganella-Gazza & $\mathrm{Ca}$ \\
PS1250 & 69 & $18 / 10$ & Pale di S.Martino & $\mathrm{Ca}$ \\
PS1255 & $69 ; 81$ & $20 / 9$ & Pale di S.Martino & $\mathrm{Ca}$ \\
PS1880 & $9 ; 47 ; 52 ; 54 ; 76$ & $18 / 8$ & Pale di S.Martino & $\mathrm{Ca}-\mathrm{Cr}$ \\
SC0250 & $14 ; 23 ; 24 ; 25 ; 28 ; 61 ; 83$ & Sette Comuni & $\mathrm{Ca}$ \\
SL1724 & 41 & $28 / 6$ & Sella & $\mathrm{Ca}$ \\
VF0745 & $16 ; 30 ; 69$ & $9 / 8$ & Vate Feltrine & $\mathrm{Ca}$ \\
VZ1178 & $12 ; 23 ; 24 ; 42 ; 57 ; 82 ; 83$ & $20 / 9$ & $\mathrm{Ca}$ \\
\hline
\end{tabular}

The two long-term survey springs were analyzed separately and were excluded from the statistical analysis.

All taxa were identified to species level, except for the Rhithrogena species-groups (hybrida and loyolaea) and Ecdyonurus gr. helveticus, because of poor specimen preservation, and Rhyacophila sensu strictu (Waringer \& Graf 1997), due to the lack of adult specimens (taxonomic keys do not identify larval instars of this group to species level).

To test for significant differences among the seven spring types, we used one-way ANOVA on $\log (\mathrm{x}+1)$ transformed abundance $(\mathrm{N})$ and taxonomic richness $(\mathrm{S})$ data, and on the Shannon-Wiener diversity index calculated from non-transformed data. Prior to analysis, we checked for normality using a Shapiro-Wilk Test, and for homoscedasticity (homogeneity of variance) using a Levene and Cochran Test. Subsequently, the Tukey post-hoc Test was used on those variables that were significantly different to find which pairs of spring types were significantly different. We performed a Canonical Correlation analysis (CANCOR) to identify any existing correlation between abundance, species richness and the environmental variables that mostly contribute to separate the spring group types (altitude, $\mathrm{pH}$, shading, current velocity, discharge, substratum composition).

A NMDS (non-metric multidimensional scaling) analysis (Clarke \& Gorley 2006) of the EPT taxa assemblages was performed to highlight possible patterns based on the spring typologies, after $\log (x+1)$ transformation, using a Bray-Curtis similarity resemblance matrix. Outliers (6 springs) were removed from the ordination to reduce the stress (goodness of fit of the regression) of the analysis.

Differences in EPT assemblage patterns in the different spring typologies were tested by running an Analysis of Similarities (ANOSIM) based on the BrayCurtis distance matrix (one-way ANOSIM, factor: spring typology). A SIMPER (Similarity Percentage) analysis was performed to detect how much two groups of springs differed, and which taxa contributed to the similarity within groups and dissimilarity between groups.

Analyses were performed using Primer 6 ver. 6.1.6 (Clarke \& Gorley 2006), Statistica ver. 8.1 (StatSoft Inc. 2008).

\section{RESULTS}

\subsection{EPT taxa distribution}

For the faunistic analyses we considered all 90 springs for which EPT taxa were identified, 88 taxa in total. Highest species richness was recorded among the Trichoptera (52 taxa), followed by the Plecoptera (39) and Ephemeroptera (6). All identified taxa are listed in table 2 .

The EPT faunistic results from the CRENODAT project were compared to the known distribution of the Italian fauna (Stoch 2000-2005). 
Tab. 2. EPT species list of the 90 springs sampled in the CRENODAT project. $\mathrm{n} .=$ identification number of species, Ab. $=$ abundance $\left(\right.$ ind $\mathrm{m}^{-2}$ ), Fr. = frequency in springs, $\mathrm{Ca}=$ relative frequency in carbonate springs, $\mathrm{Ca}-\mathrm{Cr}=$ relative frequency in carbonate-crystalline springs, $\mathrm{Cr}=$ relative frequency in crystalline springs, elev. = relative frequency in springs above $1300 \mathrm{~m}$ a.s.l., min. elev. $=$ minimum elevation of colonised spring, max. elev. $=$ maximum elevation of colonized spring, $*=$ new for Trentino, according to the Italian check list (Stoch, 2000-2005).

\begin{tabular}{|c|c|c|c|c|c|c|c|c|c|}
\hline $\mathrm{n}$. & Species & $\mathrm{Ab}$. & Fr. & $\mathrm{Ca}$ & $\mathrm{Ca}-\mathrm{Cr}$ & $\mathrm{Cr}$ & elev. & min. elev. & max. elev. \\
\hline & Ephemeroptera & & & & & & & & \\
\hline 1 & Baetis alpinus (PICTET, 1843) & 48 & 10 & 50 & 20 & 30 & 30 & 335 & 2056 \\
\hline 2 & Baetis rhodani (PICTET, 1843) & 37 & 12 & 50 & & 50 & 50 & 170 & 2153 \\
\hline 3 & Ecdyonurus gr. helveticus & 121 & 13 & 77 & 8 & 15 & 31 & 170 & 1400 \\
\hline 4 & Rhitrogena gr. hybrida & 9 & 4 & 50 & & 50 & 75 & 470 & 2153 \\
\hline 5 & Rhitrogena gr. loyolaea & 17 & 6 & 33 & & 67 & 83 & 470 & 2153 \\
\hline \multirow[t]{2}{*}{6} & Serratella ignita (PODA, 1761) & 10 & 4 & 50 & & 50 & 75 & 170 & 2153 \\
\hline & Plecoptera & & & & & & & & \\
\hline 7 & Amphinemura sulcicollis (STEPHENS, 1836) & 15 & 3 & 67 & 33 & & & 420 & 658 \\
\hline 8 & Capnia vidua* KLAPÁLEK, 1904 & 1 & 1 & & 100 & & & 586 & 586 \\
\hline 9 & Dictyogenus alpinus (PICTET, 1842) & 32 & 3 & 67 & 33 & & 67 & 1077 & 1880 \\
\hline 10 & Dictyogenus fontium (RIS, 1896) & 18 & 6 & 67 & & 33 & 67 & 950 & 2051 \\
\hline 11 & Dinocras ferreri (PICTET, 1841) & 26 & 3 & 100 & & & & 170 & 972 \\
\hline 12 & Isoperla rivulorum (PICTET, 1842) & 11 & 6 & 67 & 17 & 17 & 33 & 656 & 1379 \\
\hline 13 & Leuctra alpina KUEHTREIBER, 1934 & 1 & 1 & & & 100 & 100 & 1300 & 1300 \\
\hline 14 & Leuctra braueri KEMPNY, 1898 & 241 & 21 & 71 & 5 & 24 & 33 & 250 & 2051 \\
\hline 15 & Leuctra helvetica AUBERT, 1956 & 6 & 2 & 50 & & 50 & 100 & 1300 & 1358 \\
\hline 16 & Leuctra inermis KEMPNY, 1899 & 7 & 2 & 50 & & 50 & 50 & 656 & 1300 \\
\hline 17 & Leuctra major BRINCK, 1949 & 14 & 11 & 36 & & 64 & 82 & 720 & 1990 \\
\hline 18 & Leuctra moselyi MORTON, 1929 & 51 & 2 & 50 & 50 & & & 735 & 905 \\
\hline 19 & Leuctra niveola* SCHMID, 1947 & 1 & 1 & & & 100 & 100 & 1300 & 1300 \\
\hline 20 & Leuctra rauscheri AUBERT, 1957 & 3 & 1 & 100 & & & 100 & 1358 & 1358 \\
\hline 21 & Leuctra rosinae KEMPNY, 1900 & 1 & 1 & 100 & & & 100 & 1358 & 1358 \\
\hline 22 & Leuctra teriolensis KEMPNY, 1900 & 9 & 1 & 100 & & & 100 & 1605 & 1605 \\
\hline 23 & Nemoura cinerea (RETZIUS, 1783) & 157 & 13 & 46 & 8 & 46 & 69 & 250 & 1853 \\
\hline 24 & Nemoura flexuosa AUBERT, 1949 & 38 & 13 & 77 & & 23 & 54 & 250 & 2240 \\
\hline 25 & Nemoura mortoni (RIS, 1902) & 178 & 25 & 46 & 15 & 39 & 48 & 250 & 2153 \\
\hline 26 & Nemoura (cf.) obtusa (RIS, 1902) & 2 & 1 & & 100 & & 100 & 1435 & 1435 \\
\hline 27 & Nemoura sinuata (RIS, 1902) & 176 & 30 & 55 & 13 & 32 & 37 & 420 & 2153 \\
\hline 28 & Nemurella pictetii KLAPÁLEK, 1900 & 37 & 6 & 67 & & 33 & 50 & 250 & 2182 \\
\hline 29 & Perlodes microcephalus (PICTET, 1833) & 4 & 3 & 33 & & 67 & 100 & 1300 & 1990 \\
\hline 30 & Protonemura auberti ILLIES, 1954 & 16 & 7 & 71 & & 29 & 29 & 745 & 2153 \\
\hline 31 & Protonemura brevistyla (RIS, 1902) & 11 & 3 & 68 & & 33 & 67 & 470 & 2153 \\
\hline 32 & Protonemura caprai* (AUBERT, 1954) & 1 & 1 & & & 100 & & 1254 & 1254 \\
\hline 33 & Protonemura intricata (RIS, 1902) & 3 & 2 & 50 & & 50 & 100 & 1358 & 2153 \\
\hline 34 & Protonemura lateralis (PICTET, 1836) & 15 & 6 & 33 & 17 & 50 & 50 & 474 & 1990 \\
\hline 35 & Protonemura nimborum (RIS, 1902) & 48 & 17 & 41 & 24 & 35 & 35 & 453 & 2153 \\
\hline \multirow[t]{2}{*}{36} & Protonemura (cf.) nitida (PICTET, 1836) & 1 & 1 & & & 100 & 100 & 686 & 686 \\
\hline & Trichoptera & & & & & & & & \\
\hline 37 & Acrophylax zerberus* BRAUER, 1867 & 4 & 3 & & 33 & 67 & 100 & 1670 & 2792 \\
\hline 38 & Agapetus fuscipes CURTIS, 1834 & 6 & 1 & & 100 & & & 1077 & 1077 \\
\hline 39 & Agapetus nimbulus MCLACHLAN, 1879 & 1 & 1 & & 100 & & & 420 & 420 \\
\hline 40 & Allogamus hilaris* (MCLACHLAN, 1876) & 2 & 1 & & & 100 & 100 & 1300 & 1300 \\
\hline 41 & Allogamus uncatus (BRAUER, 1857) & 44 & 12 & 42 & & 58 & 75 & 804 & 2153 \\
\hline 42 & Chaetopteryx fusca BRAUER, 1857 & 12 & 7 & 29 & & 71 & 57 & 250 & 1990 \\
\hline 43 & Consorophylax consors* (MCLACHLAN, 1880) & 4 & 3 & 25 & & 75 & 67 & 1215 & 2153 \\
\hline 44 & Crunoecia irrorata* (CURTIS, 1834) & 10 & 6 & 50 & 33 & 17 & 17 & 430 & 1421 \\
\hline 45 & Crunoecia (cf.) kempnyi * MORTON, 1901 & 4 & 3 & 100 & & & 33 & 509 & 1315 \\
\hline 46 & Cryptothrix nebulicola MCLACHLAN, 1867 & 3 & 1 & 100 & & & 100 & 1358 & 1358 \\
\hline 47 & Diplectrona (cf.) felix * MCLACHLAN, 1878 & 79 & 16 & 63 & 25 & 13 & 13 & 453 & 1880 \\
\hline 48 & Drusus annulatus MCLACHLAN, 1878 & 27 & 1 & 100 & & & 100 & 1358 & 1358 \\
\hline 49 & Drusus (cf.) biguttatus (PICTET, 1834) & 1 & 1 & 100 & & & 100 & 1358 & 1358 \\
\hline 50 & Drusus chrysotus (RAMBUR, 1842) & 1 & 1 & & & 100 & 100 & 1685 & 1685 \\
\hline 51 & Drusus destitutus KOLENATI, 1848 & 38 & 6 & 17 & & 83 & 100 & 1353 & 2792 \\
\hline 52 & Drusus discolor (RAMBUR, 1842) & 70 & 5 & 40 & 20 & 40 & 100 & 1350 & 1990 \\
\hline 53 & Drusus monticola MCLACHLAN, 1876 & 1 & 1 & & & 100 & 100 & 1685 & 1685 \\
\hline 54 & Ecclisopteryx (cf.) asterix * MALICKY, 1979 & 16 & 5 & 20 & 20 & 60 & 60 & 656 & 2153 \\
\hline 55 & Ernodes articularis (PICTET, 1834) & 1 & 1 & 100 & & & & 420 & 420 \\
\hline 56 & Halesus rubricollis (PICTET, 1834) & 18 & 4 & 33 & & 67 & 100 & 1300 & 2792 \\
\hline
\end{tabular}


Tab. 2. Continuation.

\begin{tabular}{|c|c|c|c|c|c|c|c|c|c|}
\hline n. & Species & Ab. & Fr. & $\mathrm{Ca}$ & $\mathrm{Ca}-\mathrm{Cr}$ & $\mathrm{Cr}$ & elev. & min. elev. & max. elev. \\
\hline 57 & Lithax niger (HAGEN, 1859) & 42 & 17 & 22 & 11 & 67 & 65 & 950 & 2153 \\
\hline 58 & Melampophylax (cf.) melampus* (MCLACHLAN,1876) & 17 & 4 & 50 & 25 & 25 & 50 & 586 & 1358 \\
\hline 59 & Metanoea rhaetica SCHMID, 1955 & 8 & 1 & 100 & & & 100 & 1358 & 1358 \\
\hline 60 & Micropterna sequax MCLACHLAN, 1875 & 1 & 1 & & & 100 & 100 & 2153 & 2153 \\
\hline 61 & Odontocerum albicorne (SCOPOLI, 1769) & 59 & 12 & 58 & 25 & 17 & 8 & 170 & 1950 \\
\hline 62 & Parachiona picicornis (PICTET, 1834) & 1 & 1 & 100 & & & 100 & 1350 & 1350 \\
\hline 63 & Philopotamus ludificatus MCLACHLAN, 1878 & 7 & 1 & & & 100 & & 1200 & 1200 \\
\hline 64 & Plectrocnemia brevis MCLACHAN, 1871 & 1 & 1 & & & 100 & & 962 & 962 \\
\hline 65 & Plectrocnemia conspersa (CURTIS, 1834) & 4 & 2 & 50 & & 50 & 50 & 981 & 1944 \\
\hline 66 & Plectrocnemia geniculata MCLACHLAN, 1871 & 14 & 5 & 80 & 20 & & 20 & 510 & 1527 \\
\hline 67 & Potamophylax (cf.) cingulatus (STEPHENS, 1837) & 5 & 3 & 33 & & 67 & 67 & 250 & 1665 \\
\hline 68 & Pseudopsilopteryx zimmeri* (MCLACHLAN, 1876) & 15 & 8 & 25,0 & & 75,0 & 75 & 854 & 2792 \\
\hline 69 & Ptilocolepus granulatus (PICTET, 1834) & 5 & 4 & 75 & & 25 & 25 & 745 & 1790 \\
\hline 70 & Rhyacophila bonaparti SCHMID, 1947 & 2 & 2 & 50 & 50 & & & 1077 & 1240 \\
\hline 71 & Rhyacophila (cf.) dorsalis (CURTIS, 1834) & 5 & 1 & 100 & & & 100 & 1358 & 1944 \\
\hline 72 & Rhyacophila glareosa MCLACHLAN, 1867 & 1 & 1 & & & 100 & 100 & 1300 & 1300 \\
\hline 73 & Rhyacophila hirticornis MCLACHLAN, 1879 & 1 & 1 & & & 100 & 100 & 1300 & 1300 \\
\hline 74 & Rhyacophila intermedia MCLACHLAN, 1868 & 3 & 2 & 100 & & & 50 & 470 & 1358 \\
\hline 75 & Rhyacophila (cf.) obliterata * MCLACHLAN, 1863 & 4 & 3 & 100 & & & 67 & 565 & 1665 \\
\hline 76 & Rhyacophila (cf.) producta $*$ MCLACHLAN, 1879 & 40 & 12 & 64 & 9 & 27 & 67 & 930 & 2792 \\
\hline 77 & Rhyacophila pubescens PICTET, 1834 & 19 & 5 & 60 & 40 & & 20 & 658 & 1871 \\
\hline 78 & Rhyacophila stigmatica (KOLENATI, 1859) & 1 & 1 & 100 & & & 100 & 1436 & 1436 \\
\hline 79 & Rhyacophila tristis PICTET, 1834 & 12 & 3 & 67 & & 33 & 67 & 1200 & 1605 \\
\hline 80 & Rhyacophila (cf.) vulgaris PICTET, 1834 & 76 & 7 & 33 & 17 & 50 & 57 & 565 & 2056 \\
\hline 81 & Sericostoma personatum (KIRBY \& SPENCE, 1876) & 24 & 11 & 73 & 27 & & & 474 & 1255 \\
\hline 82 & Silo nigricornis (PICTET, 1834) & 3 & 3 & 33 & & 67 & & 633 & 1178 \\
\hline 83 & Synagapetus dubitans MCLACHLAN, 1879 & 26 & 8 & 75 & & 25 & 38 & 250 & 1474 \\
\hline 84 & Synagapetus (cf.) krawanyi * (ULMER, 1838) & 4 & 2 & 100 & & & & 656 & 1160 \\
\hline 85 & Tinodes dives (PICTET, 1834) & 1 & 1 & & & 100 & 100 & 1421 & 1421 \\
\hline 86 & Tinodes maclachlani* KIMMINS, 1966 & 10 & 1 & 100 & & & 100 & 1605 & 1605 \\
\hline 87 & Tinodes pallidulus* MCLACHLAN, 1878 & 4 & 1 & 100 & & & 0 & 930 & 930 \\
\hline 88 & Wormaldia occipitalis* (PICTET, 1834) & 1 & 1 & 100 & & & & 510 & 510 \\
\hline
\end{tabular}

Fifteen of the Trichoptera and three of Plecoptera species were new for the Trentino province, but known from other areas of the Italian Alps.

With respect to the altitudinal range, nine EPT species were found above their previously known altitudinal limit. Among these were the Trichoptera, Ecclisopteryx (cf.) asterix and Diplectrona (cf.) felix, and the Ephemeroptera, Serratella ignita and Baetis rhodani, which are rhithral species also recorded from springs (Bauernfeind et al. 2002). The Trichoptera Acrophylax zerberus was also recorded at $1670 \mathrm{~m}$ a.s.l., below its previously known altitudinal range in the Italian Alps (2000-2700 m a.s.l. (Stoch 2000-2005) (Tab. 2).

The most frequent EPT taxa encountered were the Plecoptera, Nemoura sinuata, N. mortoni, Leuctra braueri and Protonemura nimborum, recorded in 30, 25, 21 and 17 springs, respectively. Lithax niger and Diplectrona (cf.) felix were more widespread Trichoptera, found in 17 and 16 springs, respectively. The most frequent Ephemeroptera taxa were Ecdyonurus gr. helveticus (present in 13 springs), Baetis rhodani (in 12 springs) and B. alpinus (in 10 springs).

The two long-term monitoring springs (AD1300 and BR1358 of the RhHA type) contributed 13 taxa, that were not found in other springs, to the CRENODAT EPT species list. These two springs are situated on the opposite sides of the Rendena Valley, at similar elevation (1330 and $1358 \mathrm{~m}$ a.s.l. respectively) and differ mainly in their geological setting: siliceous rocks for AD1300 (Adamello-Presanella Mountain Group), and carbonate strata for BR1358 (Brenta Mountain Group). AD1300 and BR1358 hosted a total of 31 and 29 EPT species, respectively. The maximum taxonomic richness was attained in AD1300 after five sampling occasions, and in BR1358 after nine (Fig. 2).

A total of 47 EPT species was recorded in the two long-term springs, but only 13 species were common; 18 were found only at AD1300 and 16 only at BR1358. None of the species found only in the carbonate spring (BR1358) were prevalent in the other short term carbonate springs.

In the same way, the species found in $\mathrm{AD} 1300$ but not in BR1358 were not more frequent in other siliceous springs than in the other carbonate ones.

Some preferences, however, appeared for few species in the dataset. For example, some taxa were more abundant in carbonate springs, such as, Leuctra braueri, recorded in 21 springs $71 \%$ of which were carbonate, Ecdyonurus gr. helveticus (13 springs, 77\% carbonate), Sericostoma personatum (11 springs, $73 \%$ carbonate), Nemoura flexuosa (13 springs, 77 carbonate) and Synagapetus dubitans (8 springs, $75 \%$ carbonate). On the other hand, some taxa favoured springs on siliceous substrata: Litax niger (present in 17 springs, 67\% siliceous) and Leuctra major (11 springs, 64\% siliceous). 


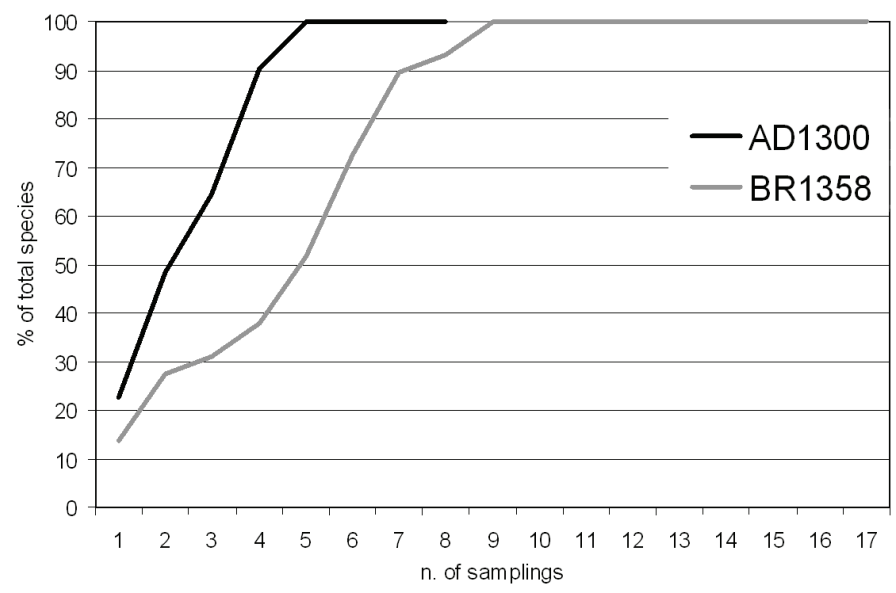

Fig. 2. EPT Species accumulation curves (\% of total species) relative to the two long-term monitored springs AD1300 (7 samplings) and BR1350 (17 samplings).

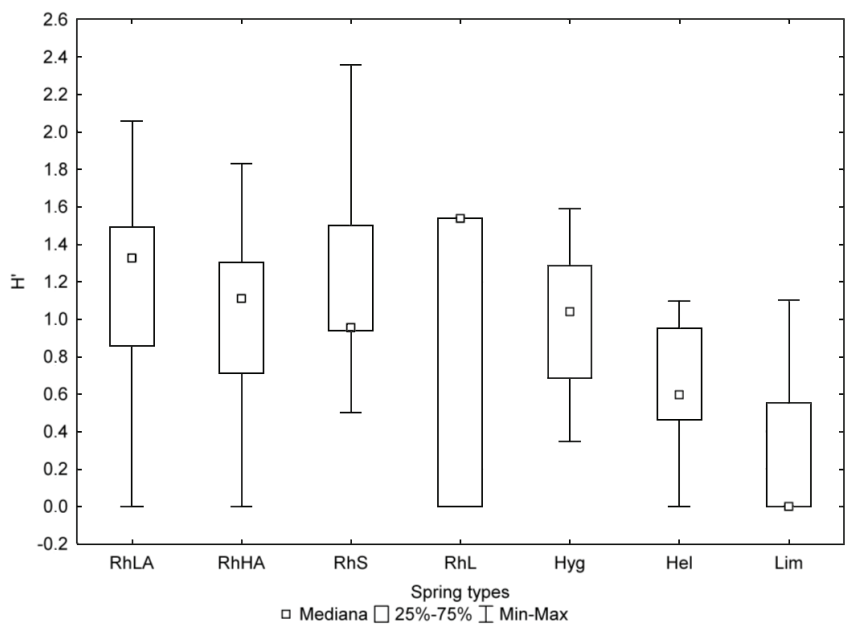

Fig. 3. Box plot of the Shannon-Wiener diversity index among spring types.

The springs were quite evenly distributed along the altitudinal range, with the median value around $1300 \mathrm{~m}$ a.s.l. (Fig. 1). We used this arbitrary threshold to separate species according to their altitudinal preferences. Of the 90 springs considered, 48 (55\%) were below and 40 $(45 \%)$ above this threshold. Of the 88 taxa, only 16 $(18 \%)$ were restricted to lower elevation springs, five (17\%) Plecoptera and 11 (21\%) Trichoptera, while 29 $(33 \%)$ were found exclusively in higher ones, $10(33 \%)$ Plecoptera and 19 (37\%) Trichoptera. Among these, Halesus rubricollis, Drusus discolor and D. destitutus were relatively common in four, five and six high elevation springs, respectively. None of the six Ephemeroptera taxa were restricted to above or below the threshold, although Rhitrogena gr. loyolaea, $R$. gr. hybrida and Serratella ignita were more widespread at higher elevations and Ecdyonurus gr. helveticus and Baetis alpinus at lower elevations. The highest spring (2792 m a.s.1) hosted only four species of Trichoptera: Acrophylax zerberus, Halesus rubricollis, Drusus destitutus and Pseudopsilopteryx zimmeri.

\subsection{Statistical analysis}

Using Anova, the Shannon-Wiener diversity index differed significantly among spring types $(F=2.3991, p$ $=0.036$; Fig. 3), with the RhL group having the highest median value. However, a significant difference was only recorded between RhLA and Lim types ( $p=$ 0.049). Taxonomic richness also differed significantly between groups $(p=0.026)$, most significantly between $\operatorname{RhLA}$ and $\operatorname{Lim}(p=0.031)$, but also significantly between RhS and Lim ( $p=0.050)$ (Fig. 4). The difference in EPT abundance was highly significant $(F=$ 3.1374, $p<0.001)$. In particular, RhLA, RhHA, RhS, and Hyg differed significantly or very significantly from Lim springs $[p=0.005 ; p=0.010 ; p=0.040 ; p=0.010$, (Fig. 5) respectively].

The CANCOR analysis results were non-significant ( $R=0.36 ; p=0.64)$. Abundance and species richness were weakly correlated with the environmental variables, i.e. altitude, $\mathrm{pH}$, shading, current velocity, dis- 


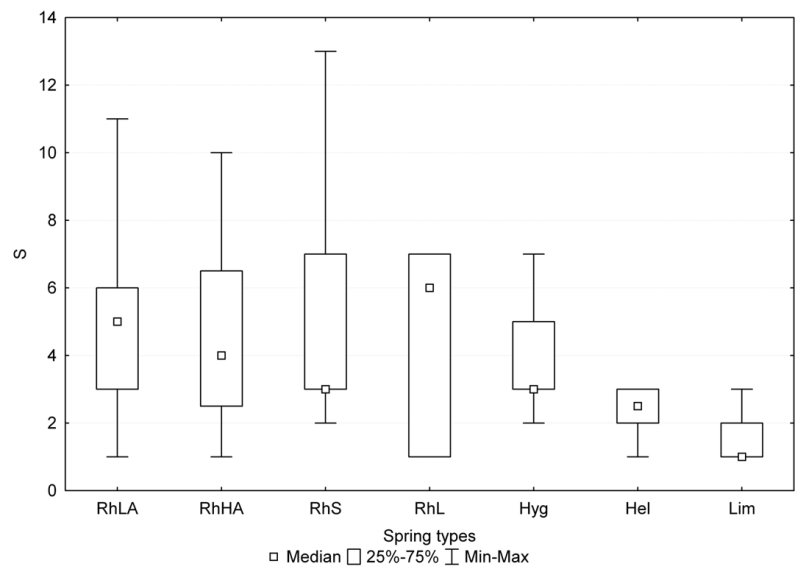

Fig. 4. Box plot of the taxonomic richness among spring types.

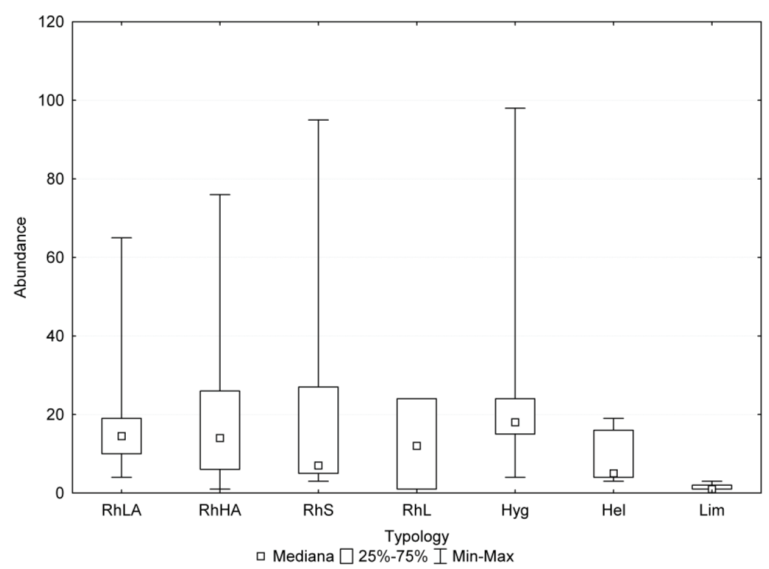

Fig. 5. Box plot of the abundances among spring types.

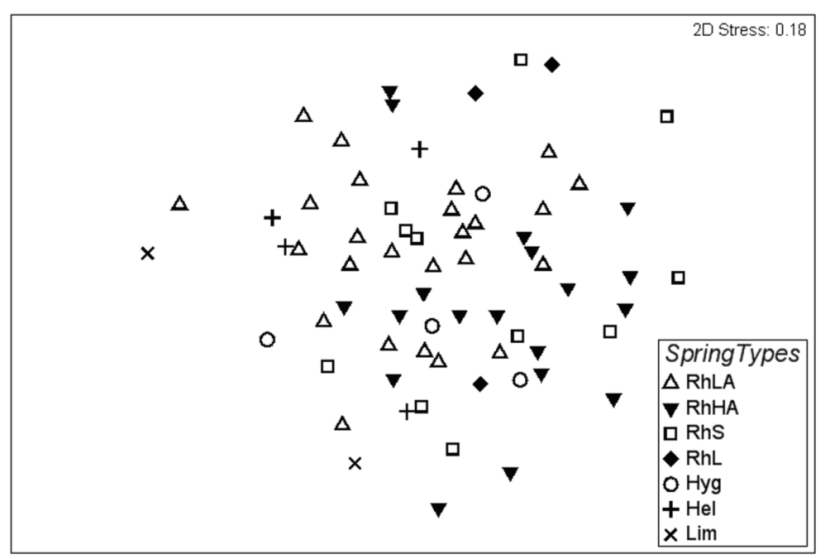

Fig. 6. NMDS (non-metric multidimensional scaling) analyses of EPT taxa assemblages according to the spring typologies.

charge, and substratum composition (Maximum correlation coefficient $R=0.16$ ).

The MDS ordination of the total invertebrate assemblage did not show a clear spatial trend in community composition (Fig. 6). However, some differences could be highlighted. The two Lim spring types (the other two were excluded as outliers) are separated from the other groups, in particular from rheocrenal groups. The $\mathrm{RhS}$ group lies mostly inside the RhHA group, which is quite clearly separated from RhLA. Hel is enclosed in RhLA, while Hyg and RhL do not seem to form clear patterns.

The one-way ANOSIM (Tab. 3) showed a significant difference in species assemblages among spring typologies $(R=0.136 ; p<0.001)$; the pairs of springs 
which had high $R$ values and differed significantly ( $p$ $<0.05)$ were: Lim $v s$ RhS, Lim vs RhLA, Lim vs RhHA, RhS vs RhLA, RhHA vs Hel and RhLA vs RhHA.

Tab. 3. ANOSIM pairwise comparison between the groups. The bold values have significant $\mathrm{R}$ and significant differences $(p<0.05)$. ANOSIM analysis is slightly significant for $R$ value $(R=0.136, p<0.001)$.

\begin{tabular}{lcc}
\hline Pairwise comparison between groups & $R$ & $p$ \\
\hline RhHA, iim & $\mathbf{0 . 3 2 7}$ & $\mathbf{0 . 0 0 3}$ \\
RhHA, Hel & $\mathbf{0 . 1 7 7}$ & $\mathbf{0 . 0 3}$ \\
RhHA, RhS & -0.028 & 0.675 \\
RhHA, RhLA & $\mathbf{0 . 0 6 1}$ & $\mathbf{0 . 0 4 3}$ \\
RhHA, RhL & -0.048 & 0.618 \\
RhHA, Hyg & 0.019 & 0.411 \\
Lim, Hel & 0.004 & 0.648 \\
Lim, RhS & $\mathbf{0 . 2 2 2}$ & $\mathbf{0 . 0 3 6}$ \\
Lim, RhLA & $\mathbf{0 . 4 4 4}$ & $\mathbf{0 . 0 0 1}$ \\
Lim, RhL & 0.111 & 0.429 \\
Lim, Hyg & -0.075 & 0.762 \\
Hel, RhS & 0.096 & 0.14 \\
Hel, RhLA & 0.159 & 0.074 \\
Hel, RhL & 0.056 & 0.345 \\
Hel, Hyg & 0.009 & 0.396 \\
RhS, RhLA & $\mathbf{0 . 2 0 2}$ & $\mathbf{0 . 0 0 3}$ \\
RhS, RhL & -0.113 & 0.821 \\
RhS, Hyg & 0.083 & 0.207 \\
RhLA, RhL & 0.202 & 0.08 \\
RhLA, Hyg & 0.175 & 0.068 \\
RhL, Hyg & 0.015 & 0.5 \\
\hline
\end{tabular}

The similarities within groups were very low (SIMPER analysis, table 4). The lowest dissimilarity was recorded for RhLA and RhS (Bray-Curtis dissimilarity: 91.58 and 94.15 respectively), followed by RhHA, RhL and Hyg springs (Bray-Curtis dissimilarity: 95.25, 97.22, 97.55 respectively). Finally, Hel showed the highest dissimilarity (97.64). Dissimilarity between spring types was mostly explained by species with low abundance.

Tab. 4. Results of SIMPER analysis; factor: spring typology. RhLA: rheocrene springs at low altitude; RhHA: rheocrene springs at high altitude; RhS: rheocrene springs on siliceous substratum; RhL: large rheocrene springs; Hyg: hygropetric springs; Hel: helocrene springs; Lim: limnocrene springs. Leubra $=$ Leuctra brueri, Rhypro = Rhyacophila (cf.) producta, Litnig = Lithax niger, Pronim = Protonemura nimborum, Nemcin = Nemoura cinerea .

\begin{tabular}{lccc}
\hline Group & Similarity \% & Main Species & Contribution \% \\
\hline RhLA & 9.30 & Leubra & 21.12 \\
RhHA & 4.75 & Rhypro & 19.10 \\
RhS & 5.85 & Litnig & 14.66 \\
RhL & 2.78 & Pronim & 100.00 \\
Hyg & 2.40 & Nemcin & 57.35 \\
Hel & 2.37 & Leubra & 70.37 \\
Lim & - & - & \\
\hline
\end{tabular}

\section{DISCUSSION}

\subsection{Species assemblages}

The springs were classified into seven groups based on the environmental variables (Spitale et al. 2011, submitted). However, the pattern of EPT species richness did not match this classification: only the limnocrene springs differed significantly from the RhLA springs. These two groups differed mainly in substratum composition (Spitale et al. 2011, submitted), which is known to be a main driver for macroinvertebrate assemblages (Ilmonen \& Paasivirta 2005; von Fumetti et al. 2006). As described by von Fumetti et al. (2006), substratum composition is paramount in determining species assemblages patterns, with discharge and water velocity acting as substratum selectors. These authors also suggest that leaf litter is one of the most important substratum components for macroinvertebrate assemblage in springs. As also observed by Ilmonen \& Paasivirta (2005), who associated limnocrene springs with low diversity, springs of this type (except for one case) supported very low EPT diversity, and the data were not suitable for statistical analysis.

The differences in abundance between some of the spring groups were significant. The higher abundance of EPT taxa in rheocrene (with the exception of RhL) and hygropetric, compared with limnocrene springs highlights the importance of discharge for the crenic fauna. These two groups differed mainly in altitude, but also in discharge, which was much higher in Lim. Altitude is generally considered to be an important factor for macroinvertebrate taxonomic richness, but not for taxon abundances (Barquín \& Death 2006). On the other hand, discharge and habitat stability (which can also be dependent on discharge), are considered as predictors of abundance and richness (Hoffsten \& Malmqvist 2000; Ilmonen \& Paasivirta 2005; Barquín \& Death 2006; von Fumetti et al. 2006). In this study, the CANCOR analyses did not identify any good predictors for EPT species richness and abundance. In fact, the correlation between these two variables and the considered environmental drivers was very low and not significant, suggesting that no clear gradient was present.

The Hyg group differed significantly in abundance from the Lim group. The two spring groups differed mainly in water velocity, higher in hygropetric springs, and discharge, higher in limnocrene springs. Limnocrene springs had very low species richness, as also reported by other authors (e.g., Ilmonen \& Paasivirta 2005).

The MDS ordination did not identify a very clear pattern in EPT spring assemblages, although some separations were highlighted. The ANOSIM analysis gave a significant result, but the $\mathrm{R}$ value was low, indicating that EPT species assemblages often overlapped (Clarke $\&$ Gorley 2006). The limnocrene springs were significantly different from most rheocrene springs. Also, as above, high discharge values in limnocrene springs 
seemed to act as substratum sorting and species assemblages drivers (von Fumetti et al. 2006). The two spring types characterized by higher discharge (Lim and $\mathrm{RhL}$ ) were not significantly different. RhS differed significantly from $\mathrm{RhLA}$, in altitude, and also $\mathrm{pH}$ and substratum (siliceous vs carbonate). However, $\mathrm{pH}$ and substratum also differed between RhS and RhHA, although they were not significantly different in their species assemblages. Consequently, altitude seemed to be the main variable separating these two groups. This agrees with Zollhöfer (1999) and Barquín \& Death (2009), who considered altitude a predictor of species richness and species composition.

Hel springs were significantly different from RhHA groups. In this case, the environmental variables which differed the most were substratum composition (mainly clay and sand in Hel, stones and rocks in RhHA), although substratum composition of Hel also differs from that of other rheocrene groups. However, these two groups were different also in the organic substratum, representing the two extreme values for the dataset (highest in Hel, lowest in RhHA); differences in organic matter can lead to significantly different habitat preferences for different species (Scarsbrook et al. 2007).

With the SIMPER analysis, we did not detect any consistent EPT species assembly pattern related to the spring typology, as shown by the low similarity recorded within each group of species. Some taxa contributed to the similarity within the springs of each group (because they were most abundant in the springs of each group) with the exception of Lim springs, for which no indicative taxon was identified. All the taxa are known as crenophiles (Bauernfeind et al. 2002; Graf et al. 2002a; Graf et al. 2002b), but not as spring-specialists (crenobiontic). No species was exclusive to one spring group.

A random pattern in EPT spring species assemblages has been reported elsewhere (Erman \& Erman 1990; Myers \& Resh 2002). Abiotic variables have not been considered good drivers of creno-coenoses (Staudacher $\&$ Füreder 2007); each spring has its own history and its own abiotic characteristics which select unique community patterns (Myers \& Resh 2002).

\subsection{Faunistic considerations}

In the Italian Alps, the diversity and assemblages of EPT taxa of springs is only known from few areas (Cantonati et al. 2006; Sambugar et al. 2006; Dumnicka et al. 2007).

Diptera, Trichoptera and Plecoptera are generally dominant in Alpine springs, while Ephemeroptera are less abundant than Plecoptera and Trichoptera (Cantonati et al. 2006; Gerecke \& Franz 2006). Ephemeroptera are rarely found in springs, with Baetis rhodani or $B$. alpinus relatively abundant where periphyton is well developed (Thorup \& Lindegaard 1977; Ilmonen et al. 2009). This was confirmed in our research, although the
Heptageniidae were abundant in several springs, particularly at high elevations. Plecoptera, especially herbivorous species, are usually recorded in crenal habitats (Ilmonen \& Paasivirta 2005). The most abundant and frequent species that we recorded belonged to the detritivore genera, Nemoura, Protonemura and Leuctra, but five predatory species were also quite frequent and abundant. Based on existing literature (e.g., Erman \& Erman 1990; 1995; Glazier 1991) our results confirm that the Trichoptera are the most species-rich EPT order in springs, although they were generally less abundant than the Plecoptera in this study.

Some species (for example the Ephemeroptera Serratella ignita and Baetis rhodani, the Plecoptera, Protonemura intricata, $P$. auberti and the Trichoptera, Rhyacophila (cf.) producta, Ecclisopteryx (cf.) asterix, Drusus destitutus and D. (cf.) felix were recorded in springs above their known altitudinal range (Stoch 2000-2005), suggesting the role of Alpine springs as refugia. In fact, due to their stable ecological features and their interface with freshwater and groundwater systems, they can support a variety of relatively rare and unusual fauna (Glazier 1991; Di Sabatino et al. 2003), providing refuge from disturbance events or extreme seasonal conditions. Furthermore, they may act as a source of downstream colonizers and a permeable ecotone for the interaction of phreatic and surface communities (Stanford \& Ward 1983).

The two springs with long term data highlight the fact that repeated sampling campaigns are necessary to approach a complete species list. Thus, it is likely that results based on a single sampling occasion could strongly underestimate species assemblages and the possible differences/similarities among springs. Furthermore, seasonal differences (Tab. 1) may also have influenced species assemblages.

Temperature is recognised as a major driver for species distribution and as highly variable in Alpine streams (Brown \& Hannah 2008). Only spot temperature readings were available for all springs, and species assemblages could not therefore be analysed in relation to parameters such as annual or seasonal degree days. This could explain the presence of species above or below their known altitudinal limits. It would be expected that low elevation crenal headwaters will have cooler summer water temperatures than surrounding streams, and that higher elevation ones will have have milder temperatures with respect to glacial-snowmelt or rain-fed streams in the same areas.

\section{CONCLUSIONS}

The analysis of EPT taxa from 90 Alpine springs confirms that EPT taxa have a rather patchy distribution in Alpine springs. Though altitude appeared to be a main factor determining species distribution, significant physicochemical drivers for EPT species assemblages were not identified. Our results indicate the important 
role of springs for regional biodiversity, as fifteen Trichoptera and three Plecoptera species were new for the Trentino, and several taxa were recorded above or below their previously known altitudinal range. The importance of long term monitoring to evaluate species assemblages of Alpine springs is emphasized.

\section{ACKNOWLEDGEMENTS}

This work was supported by the CRENODAT Project (Biodiversity assessment and integrity evaluation of springs of Trentino - Italian Alps - and long-term ecological research, 2004-2008) funded by the University and Scientific Research Department of the Autonomous Province of Trento, Italy. Special thanks are due to R. Fochetti (University of La Tuscia) and J.M. Tierno de Figueroa (University of Granada) for assistance in the identification of Plecoptera.

\section{REFERENCES}

Barquín, J. \& R.G. Death. 2006. Spatial patterns of macroinvertebrate diversity in New Zealand springbrooks and rhithral streams. J. N. Am. Benthol. Soc., 25(4): 768-786.

Barquín, J. \& R.G. Death. 2009. Physical and chemical differences in karst springs of Cantabria, northern Spain: do invertebrate communities correspond? Aquat. Ecol., 43: 445-455.

Bauernfeind, E., Moog, O. \& P. Weichselbaumer. 2002. Ephemeroptera - Part III, 24 pp., In: Moog O. (Ed.), Fauna Aquatica Austriaca, Edition 2002.-Wasserwirtschaftskataster, Bundesministerium fur Land- und Fortwirtschaft, Umwelt und Wasserwirtschaft, Wien.

Belfiore, C. 1983. Efemerotteri (Ephemeroptera) In: Ruffo, S. (Ed.), Guide per il riconoscimento delle specie animali delle acqua interne italiane, vol. 24. C.N.R.-Servizio Pubblicazioni, AQ/1/2001: 112 pp.

Bottazzi, E., M.C. Bruno, M. Mazzini, V. Pieri \& G. Rossetti. 2008. First report on Copepoda and Ostracoda (Crustacea) from northern Apenninic springs (N. Italy): a faunal and biogeographical account. J. Limnol., 67(1): 56-63.

Brown, L. E. \& D. M. Hannah. 2008. Spatial heterogeneity of water temperature across an alpine river basin. Hydrol. Process., 22: 954-967.

Cantonati, M. (Ed.). 1998. Le sorgenti del Parco AdamelloBrenta. Ricerche idrobiologiche su fonti non captate. Parco Documenti, Parco Naturale Adamello-Brenta Strembo (TN): $177 \mathrm{pp}$.

Cantonati, M., E. Bertuzzi, R. Gerecke, K. Ortler \& D. Spitale. 2005. Long-term ecological research in springs of the Italian Alps: six years of standardized sampling. Verh. Internat. Verein Limnol., 29: 907-911.

Cantonati, M., E. Bertuzzi \& D. Spitale (Eds). 2007. The spring habitat: biota and sampling methods. Museo Tridentino di Scienze Naturali, Trento. Monografie del Museo Tridentino di Scienze Naturali, 4: $350 \mathrm{pp}$.

Cantonati, M., R. Gerecke \& E. Bertuzzi. 2006. Springs of the Alps-sensitive ecosystems to environmental change: from biodiversity assessments to long-term studies. Hydrobiologia, 562: 59-96.

Cantonati, M., N. Angeli, E. Bertuzzi, D. Spitale \& H. LangeBertalot. (2011). Diatoms in springs of the Alps: Spring types, environmental determinants, and substratum. In: M. Cantonati, L. Füreder, I. Jüttner \& E.J. Cox (Eds), Ecology of Springs. Journal of the North American Benthological Society. Special Issue: (in preparation).

Cianficconi, F., C. Corallini \& G.P. Moretti. 1998. Trichopteran fauna of the Italian springs. In: Botosaneanu, L.
(Ed.), Studies in crenobiology. The biology of springs and springbrooks. Backhuys Publishers - Leiden: 125-140.

Clarke, K.R. \& R.N. Gorley. 2006. PRIMER v6: User Manual/Tutorial. PRIMER-E, Plymouth.

Consiglio, C. 1980. Plecotteri (Plecoptera). In: Ruffo S. (Ed.), Guide per il riconoscimento delle specie animali delle acque interne italiane, Vol. 9. C.N.R. -Servizio Pubblicazioni, AQ/1/77: 68 pp.

Crema, S., U. Ferrarese, D. Golo, P. Modena, B. Sambugar \& R. Gerecke. 1996. Ricerche sulla fauna bentonica ed interstiziale di ambienti sorgentizi in area alpina e prealpina. Report Centro Ecologia Alpina, 8: 106 pp.

D'Ambrosio, P., B. Fiasca \& D.M.P. Galassi. 2003. Caratterizzazione tipologica, biologica ed ambientale di sistemi sorgivi del Gran Sasso (Abruzzo). Thal. Sal., 26: 249-260.

Di Lorenzo, T., P. De Laurentiis \& D.M.P. Galassi. 2003. L'inferenza biologica nella valutazione del grado di protezione naturale di sorgenti carsiche captate. Thal. Sal., 26: 241-248.

Di Sabatino, A., B. Cicolani \& R. Gerecke. 2003. Biodiversity and distribution of water mites (Acari, Hydrachnidia) in spring habitats. Freshwat. Biol., 48, 2163-2173.

Dumnicka, E., J. Galas \& P. Koperski. 2007. Benthic invertebrates in karst springs: does substratum or location define communities? Int. Rev. Hydrobiology, 92: 452-464.

Erman, N.A., \& D.C. Erman. 1990. Biogeography of caddisfly (Trichoptera) assemblages in cold springs of the Sierra Nevada (California, USA). California Water Resources Centre Contribution 200.

Erman, N.A. \& D.C. Erman. 1995. Spring permanence, Trichoptera species richness, and the role of drought. $J$. Kans. Entomol. Soc., 68: 50-64.

Fochetti, R. \& J.M. Tierno de Figueroa 2008. Plecoptera. In: Fauna d'Italia. Vol. XLIII. Calderini Ed.: 339 pp.

von Fumetti, S., P. Nagel \& B. Baltes. 2006. Factors governing macrozoobenthic assemblages in perennial springs in north-western Switzerland. Hydrobiologia, 568: 467-475.

Galassi, D.M.P. 1997. Little known harpacticoid copepods from Italy and description of Parastenocaris crenobia $\mathrm{n}$. sp. (Copepoda, Harpacticoida). Crustaceana, 70: 694-709.

Gerecke, R. \& H. Franz (Eds). 2006. Quellen im Nationalpark Berchtesgaden. Lebensgemeinschaften als Indikatoren des Klimawandels. Nationalpark Berchtesgaden. Forschungsbericht, 51: $272 \mathrm{pp}$.

Gerecke, R., M. Cantonati, D. Spitale, E. Stur \& S. Wiedenbrug. 2011. The challenges of long-term ecological research in springs in the northern and southern Alps: indicator groups, habitat diversity, and medium-term change. In: M. Cantonati, R. Gerecke, I. Jüttner \& E.J. Cox (Eds), Springs: neglected key habitats for biodiversity conservation. J. Limnol. 70(Suppl. 1): 168-187.

Gerecke, R., C. Meisch, F. Stoch, F. Acri \& H. Franz. 1998. Eucrenon-hypocrenon ecotone and spring typology in the Alps of Berchtesgaden (Upper Bavaria, Germany). A study of microcrustacea (Crustacea: Copepoda, Ostracoda) and water mites (Acari: Halacaridae, Hydrachnellae). In: Botosaneanu, L. (Ed.), Studies in crenobiology. The biology of springs and springbrooks. Backhuys Publishers, Leiden: 167-182.

Glazier, D.S. 1991. The fauna of North American temperate cold springs: patterns and hypotheses. Freshwat. Biol., 26: 527-542.

Glazier, D.S. 1998. Springs as model systems for ecology and evolutionary biology: a case study of Gammarus minus Say (Amphipoda) in mid-Appalachian springs differing in $\mathrm{pH}$ and ionic content. In: Botosaneanu, L. (Ed.), Studies in crenobiology. Blackhuys Publishers, Leiden: 251-261.

Glazier, D.S. \& J.L. Gooch. 1987. Macroinvertebrate assemblages in Pennsylvania (U.S.A.) springs. Hydrobiologia, 150: 33-43. 
Graf, W., Grasser, U. \& J. Waringer. 2002a. Trichoptera Part III, In: Moog O. (Ed.), 2002. Fauna Aquatica Austriaca, Edition 2002. - Wasserwirtschaftskataster, Bundesministerium für Land- und Fortwirtschaft, Umwelt und Wasserwirtschaft, Wien: 42 pp.

Graf, W., Grasser, U. \& A. Weinzierl. 2002b. Plecoptera Part III, In: Moog O. (Ed.), 2002. Fauna Aquatica Austriaca, Edition 2002. - Wasserwirtschaftskataster, Bundesministerium für Land- und Fortwirtschaft, Umwelt und Wasserwirtschaft, Wien: $17 \mathrm{pp}$.

Hahn, H.J. 2000. Studies on classifying of undisturbed springs in southwestern Germany by macrobenthic communities. Limnologica, 30: 247-259.

Hoffsten, P.O. \& B. Malmqvist. 2000. The macroinvertebrate fauna and hydrogeology of springs in central Sweden. Hydrobiologia, 436: 91-104.

Ilmonen, J. \& L. Paasivirta. 2005. Benthic macrocrustacean and insect assemblages in relation to spring habitat characteristics: patterns in abundance and diversity. Hydrobiologia, 533: 99-113.

Ilmonen, J., L. Paasivirta, R. Virtanen \& T. Muotka. 2009. Regional and local drivers of macroinvertebrate assemblages in boreal springs. J. Biogeogr., 36: 822-834.

Lechthaler W. \& W. Stockinger. 2005. Trichoptera - Key to Larvae from Central Europe. CD-Rom-Edition, Vienna

Mezzanotte, E \& B. Sambugar. 2004. Contributo alla conoscenza della fauna delle sorgenti . In: Latella, L. (Ed.), Il Monte Pastello. Mem. Mus. Civ. St. Nat. Verona - 2 Monografie Nautralistiche: 283-292.

Moretti, G.P. 1983. Tricotteri (Trichoptera). In: Ruffo, S. (Ed.): Guide per il riconoscimento delle specie animali delle acque interne italiane, vol. 19. C.N.R.-Servizio Pubblicazioni, AQ/1/196: 155 pp.

Myers, M.J. \& V.H. Resh. 2002. Trichoptera and other macroinvertebrates in springs of the Great Basin: species composition, richness and distribution. West. N. Am. Naturalist., 62(1): 1-13.

Orendt, C. 2000. The chironomid communities of woodland springs and spring brooks, severely endangered and impacted ecosystems in a lowland region of eastern Germany (Diptera: Chironomidae). J. Insect Conserv., 4: 79-91.

Sambugar, B., G. Dessi, A. Sapelza, A. Stenico, B. Thaler \& A.Veneri. 2006. Fauna sorgentizia in Alto Adige. Provincia Autonoma di Bolzano: $372 \mathrm{pp}$.

Sartori, M. \& P. Landolt. 1999. Ephemeroptera Atlas. Fauna Helvetica 3, Centre Suisse de Cartographie de la Faune: $214 \mathrm{pp}$.
Scarsbrook, M., J. Barquin \& D. Gray. 2007. New Zealand coldwater springs and their biodiversity. Science for Conservation, 278: $72 \mathrm{pp}$.

Spitale, D., M. Leira, N. Angeli \& M. Cantonati. (2011). Environmental classification of springs of the Italian Alps and its consistence across multiple taxonomic groups. In: M. Cantonati, L. Füreder, I. Jüttner \& E.J. Cox (Eds), Ecology of Springs. Journal of the North American Benthological Society. Special Issue: (Submitted).

Stanford, J.A. \& J.V. Ward.1983. Insect species diversity as a function of environmental variability and disturbance in stream systems. In: Barnes, J.R. \& G.W. Minshall (Eds), Stream ecology: application and testing of general ecological theory. Plenum Press, New York: 265-278.

StatSoft, Inc. 2008. STATISTICA (data analysis software system), version 8.0. www.statsoft.com.

Staudacher, K. \& L. Füreder. 2007. Habitat complexity and invertebrates in selected alpine springs (Schütt, Carinthia, Austria). Int. Rev. Hydrobiol., 92: 465-479.

Stoch, F. 2000-2005. CKmap for Windows. Version 5.1. Ministero dell'Ambiente e della Tutela del Territorio, Direzione per la Protezione della Natura. http://ckmap.faunaitalia.it.

Stoch, F., \& G. Tomasin. 2002. La fauna delle acque carsiche sotterranee del Montello. Speleologia Veneta, 10: 41-70.

Stoch, F., D. Valenti, M. Chiesi \& G. Tomasin. (2011). Monitoraggio biologico delle sorgenti salse di Poiano (Reggio Emilia). Memorie dell'Istituto Italiano di Speleologia: (in press).

Thorup, J. \& C. Lindegaard.1977. Studies on Danish springs. Folia limnol. Scand., 17: 7-15.

Ward, J.V. \& K. Tockner. 2001. Biodiversity: Towards a unifying theme for river ecology. Freshwat. Biol., 46: 807819 .

Waringer, J. \& W. Graf. 1997. Atlas der Österreichischen Köcherfliegenlarven, unter Einschluss der angrenzenden Gebiete. Vienna, Facultas Universitätsverlag: 286 pp.

Williams, D.D. \& N.E. Williams. 1998. Invertebrate communities from freshwater springs: what can they contribute to pure and applied ecology? In: Botosaneanu, L. (Ed.), Studies in crenobiology. Blackhuys Publishers, Leiden: 251-261.

Zollhöfer, J.M. 1999. Spring biotopes in northern Switzerland: Habitat heterogeneity, zoobenthic communities and colonization dynamics. Doctoral thesis, Swiss Federal Institute of Science and Technology, Zürich, Switzerland: 138 pp. 